(Aus dem Institut für allgemeine und experimentelle Pathologie in Wien. [Vorstand: Hofrat Prof. Dr. Rich. Paltauf].)

\title{
Beiträge zur pathologischen Histologie der ansteckenden Blutarmut der Pferde.
}

\author{
Von \\ Dr. R. Hermann Jaffé, \\ Assistent des Institats. \\ Mit 3 Textabbildungen. \\ (Eingegangen am 19. Mai 1921.)
}

Die Widersprüche, denen wir heute noch in der Auffassung der Pathogenese der menschlichen perniziösen Anämie begegnen, die dunkle Ätiologie vieler Fälle lenken die Aufmerksamkeit auf ähnliche Erkrankungen der Tiere, in der Voraussetzung, durch vergleichende Untersuchungen neue Richtlinien für die Beurteilung der strittigen Fragen gewinnen zu können. Aus diesem Grunde wurden die experimentell durch Blutgifte erzeugten Anämien so eingehend studiert. Von größerem Wert sind die Untersuchungsergebnisse bei spontanen Erkrankungen, da hier die Verhältnisse natürlicher lieger als im willkürlich gewählten Experiment. In den letzten Jahren hat nun eine Krankheit der Pferde besondere Beachtung gefunden, die früher nur in einzelnen Gebieten Europas vorkam, während des Krieges an den meisten Fronten festgestellt wurde und später in verschiedenen Gegenden Mitteleuropas auftrat, oft vermutlich durch aus der Kriegsverwendung entlassene Pferde eingeschleppt. Unter dem Namen, mit denen diese Krankheit bezeichnet wird, findet sich auch der Ausdruck ,,perniziöse Anämie der Pferde" und es hat nicht an Versuchen gefehlt, Beziehungen zwischen ihr und der gleichbenannten Krankheit der Menschen aufzustellen [vor allem R. Seyderhelm ${ }^{1}$ ) und Stroh ${ }^{2}$ )]. Doch sehen wir, daß die Anämie bei der Erkrankung der Pferde keineswegs konstant in den Vordergrund der Erscheinungen tritt, daß ein typisches perniziös-anämisches Blutbild kaum je zur vollen Ausbildung gelangt und da $B$ es gar nicht so selten Fälle gibt, bei denen bis zum Tode fast normale Blutwerte gefunden

1) Seyderhelm; Arch. f. exp. Pathol. v. Pharmakol. r6. 1914; Zieglers Beiträge z. allg. Path. u. pathol. Ana . 58. 1914. 285; Dtsch. Arch. f. klin. Med. 126, 95. 1918 (Literaurt).

2) Stroh, Münch. tierärztl. Wochenschr. 68, Nr.47. 1917. 
werden. Das gilt insbesondere von der akuten Form. Das Hauptgewicht wird daher von anderer Seite auf die Infektiosität gelegt, die in den Fieberanfällen und vor allem in der Übertragbarkeit durch minimale Mengen filtrierten Serums zum Ausdrucke kommt. Aus diesem Grunde wird von vielen Autoren die von den Franzosen eingeführte Bezeichnung ,,infektiöse Anämie" vorgezogen und manche, wie Lührsi), wollen überhaupt den Ausdruck Anämie fallen lassen und sprechen von einem ansteckenden, rekurrierenden Fieber ${ }^{2}$ ).

Diagnostisch scheint dieses rekurrierende Fieber beträchtliche Schwierigkeiten zu bereiten. Bei der Unkenntnis des Erregers, der in die Gruppe der invisiblen Virus gehören soll, und bei dem wenig charakteristischen Blutbild ist zur. Sicherung der Diagnose der geglückte Übertragungsversuch auf ein gesundes Pferd notwendig. Die Obduktion bietet nach den übereinstimmenden Schilderungen keinen besonderen Befund (Hutyra und Marek ${ }^{3}$ ), Seyderhelm ${ }^{4}$ ), Schebitz ${ }^{5}$ ), Lührs (1. e.), Reinhardt ${ }^{6}$ ) u. a.]. Es sind die anatomischen Veränderungen einer akuten resp. chronischen Septikämie. Meist ist ein Milztumor vorhanden, die großen drüsigen Organe zeigen Schwellung, verschiedene Grade degenerativer Veränderungen, dann finden sich seröse Ergüsse in den Körperhöhlen, succulente Schwellung und Hyperämie der Lymphknoten, Blutungen in den serösen Häuten, in der Leber, in der Niere und im Knochenmark.

Unter den histologischen Befunden sind in erster Linie die Mitteilungen von Seyderhelm (l.c.) und Stroh (l. c.) hervorzuheben. Sie beschrieben bei den chronischen Fällen eine hochgradige myeloide Metaplasie der Leber und der Milz. Die extramedullären Blutbildungsherde glichen denen bei Pferdeembryonen und damit war eine Brücke zur perniziösen Anämie des Menschen geschlagen, bei der das Wiedererwachen embryonaler Myelopoesie in der Leber und in der Milz zuerst von Me yer und Heine ke betont wurde. Neben den Zeichen gesteigerter Blutbildung fanden sich solche gesteigerten Blutzerfalles in Form von Ablagerungen eisenhältigen Blutpigmentes in der Leber und in der Milz (Seyderhelm, Stroh, Lührs).

Die Fälle, die meinen Ausführungen zugrunde liegen, entstammen der Mödlinger Tierimpfstoffgewinnungsanstalt und ich bin deren Leiter Herrn Doz. Dr. Gerlach für die Überlassung des Materials zu großem

1) Lührs, Zeitschr. f. Veterinärk. 31. 1919.

$\left.{ }^{2}\right) \mathrm{Ob}$ es daneben bei Tieren noch eine echte perniziöse Anämie gibt, ist. zweifelhaft (Hutyra und Marek).

3) Hutyra und Marek, Spez. Pathologie und Therapie der Haustiere. I. Bd., S. 849.1910 .

4) Seyderhel m, Zieglers Beiträge z. allg. Path, u. pathol. Anat. 58. 1914.

$\left.{ }^{5}\right)$ Schebitz, Berl. tierärztl. Wochenschr. 1918, S. 82.

6) Reinhardt, Monatshefte f. prakt. Tierheilk. 29, 526.1919. 
Danke verptlichtet. Über die Vorgeschichte der Fälle kann ich mich kurz fassen. Es handelte sich um anscheinend ganz gesunde Pferde, die früher in militärischer Verwendung gestanden hatten. Bei den täglichen Temperaturmessungen fielen bei diesen Pferden in kürzeren oder längeren Intervallen auftretende Fieberanfälle auf, für die kein ersichtlicher Grund vorhanden war. Der Verdacht auf infektiöse Anämie wurde durch den positiven Ausfall des Übertragungsversuches bestätigt. Die Anämie war nie besonders hochgradig, als niedrigste Hämoglobinwerte notierten wir 35-40\% (Sahli). In den Ausstrichpräparaten war meistens eine Anisocytose angedeutet, Poikilocytose, Polychromasie und basophile Punktierung wurden vermißt [Seyderhelm (l. c.), Wirth' ${ }^{1}$ ), Fröhner ${ }^{2}$ ) u. a.]. Bei mehreren Tieren fanden sich spärliche Normoblasten. Sie zeigten mitunter Basophilie des Protoplasmas und Zerteilung der Kerne in mehrere, vërschieden große Kugeln, wodurch bisweilen recht eigenartige, an Parasiten erinnernde Bilder entstanden. Megaloblasten ließen sich in keinem Präparate nachweisen. Das Verhalten der weißen Blutkörperchen war nicht konstant. Bei den chronischen Fällen lag eine mäßige Verminderung ihrer Zahl vor. Deutlicher war die oft betonte, relative Lymphocytose. Die durchschnittlichen Werte lauteten: Lymphocyten 40-62\%, polymorphkernige Leukocyten 14-40\%, Monocyten 4-6\%, Eosinophile 2-6\%. Nur in einem Falle fehlten die Eosinophilen vollkommen. Bei akutem Verlaufe (Impf-Pferde) argab die Differentialzählung: Lymphocyten $8-21 \%$, polymorphkernige Leukocyten $64-80 \%$, Monocyten $3-8 \%$, Eosinophile $1 / 2-2 \%$. Schließlich wäre noch die ausgesprochene Verminderung der Blutplättchen hervorzuheben.

Die spontan erkrankten und die geimpften. Pferde wurden nach kürzerer oder längerer Beobachtung geschlachtet. Die Schlachtbefunde stimmten vollkommen mit den oben zitierten Angaben überein. Verendete Pferde standen mir nicht zur Verfügung, doch glaube ich nicht, da $B$ sich die Befunde bis zum spontanen Ende noch wesentlich geändert hätten, zumal in manchen Fällen die Kachexie schon so weit fortgeschritten war, daß die Tiere wegen derselben und wegen der Lähmung der Hinterhand zur Schlachtung gefahren werden mußten.

Die histologischen Befunde sollen gesondert nach der Dauer der Erkrankung besprochen werden. Durch Vergleich der akuten Fälle mit den chronischen werden sich dann leichter die bei letzteren gefundenen Veränderungen deuten lassen. Als akute Fälle bezeichne ich die Impf-Pferde. Der Zwischenraum zwischen Impfung $(5 \mathrm{ccm}$ Serum subcutan) und erstem Fieberanfall betrug 6-14 Tage. Nach 1-3 Fieberanfällen wurden die Tiere getötet (Krankheitsdauer 12-21 Tage).

1) Wirth, Monatshefte f. prakt. Tierheilk. 29, Heft 3/4.

2) Fröhner, ibidem S. 384 . 
Über die Krankheitsdauer der chronischen Fälle kann ich keine sicheren Angaben machen. Die Tiere standen $1 / 2$ bis 2 Jahre in Beobachtung. Die Milz ist im akuten Stadium stets beträchtlich vergrößert. Auf der Schnittfläche quillt die breiig erweichte, dunkelrote Pulpa vor, die normale Organzeichnung verwischend. Öfters finden sich, namentlich an den Bändern, größere Blutungen. - Im mikroskopischen Präparate fällt in erster Linie der enorme Blutreichtum der erweiterten Sinus auf. Die roten Blutkörperchen liegen dicht aneinandergepreßt, lassen sich aber noch gut voneinander abgrenzen. Zahlreiche Erythrocyten finden sich auch in den Pulpasträngen, wo sie von Makrophagen aufgenommen werden. Zunächst sind die phagocytierten roten Blutkörperchen noch gut erkennbar, später verwandeln sie sich allmählich in braune Pigmentschollen. Auch weiße Blutkörperchen und deren Überreste in Form dunkler Kernbröckel findet man in den großen, phagocytierenden Zellen. An manchen Stellen trifft man auf größere Blutaustritte. Zwischen den Makrophagen und Erythrocyten liegen kleinere und größere lymphoide Elemente mit schmalem basophilem Protoplasma, das keine Oxydasereaktion gibt, ferner in wechselnder Menge Plasmazellen und sehr reichlich, bisweilen in enormer Zahl eosinophile Leukocyten. Sie bilden oft, besonders am Rand von Follikeln große Haufen und besitzen meist reife, segmentierte, seltener runde, helle Kerne. Oft kann man deutlich das Hindurchtreten eosinophiler Zellen durch die Wand erweiterter Pulpavenen verfolgen. Normoblasten sah ich nur vereinzelt. Sie zeigten die gleichen Zerschnürungen der Kerne, wie ich sie im peripheren Blute beschrieben habe. Auch sie wurden von Histiocyten aufgenommen. Knochenmarksriesenzellen konnte ich nur bei einem jüngeren Pferde nachweisen. Die Malpighischen Körperchen erschienen aufgelockert, sonst unverändert, Keimzentra ließen sich nicht unterscheiden. Nur einmal waren die Follikel bis auf geringe Reste geschwunden.

Bei längerer Krankheitsdauer wird die Milz derber. Sie ist aber auch weiterhin in der Regel vergrößert (Gewicht 3-8 kg). Die Schnittfläche ist jetzt braunrot und weist unregelmäßige Vorwölbungen auf, die Follikel sind als kleine, blaßgraue Fleckchen zu erkennen. Das histologische Bild wird von den oft ungeheueren Massen eisenhältigen Blutpigmentes beherrscht, das in Form kleiner Körner und grober Schollen die anderen Elemente der Pulpa zu verdrängen scheint und nur die Follikel freiläßt. Eine richtige Vorstellung von den Pigmentmengen bekommt man bei der Turnbullblaureaktion, bei der die Pulpastränge sich fast gleichmäßig leuchtend blau tingieren. Das Pigment liegt.zum größten Teil intracellulär, von großen Wanderzellen aufgenommen. Seltener trifft man Makrophagen mit erhaltenen Erythrocyten, öfters solche, die durch das Abblassen der aufgenommenen

Virchows Archiv. Bd. 233. 
Erythrocyten vakuolisiert erscheinen. Zwischen den pigmentführenden Zellen finden sich lymphoide Zellformen, Plasmazellen, vereinzelte Normoblasten und sehr viele eosinophile Leukocyten. Myelocyten sind spärlich und vorwiegend acidophil gekörnt, Riesenzellen kommen nur in einzelnen Exemplaren vor. Den breiten, zellreichen Pulpasträngen gegenüber erscheinen die Sinus schmal und zellarm. Die Follikel sind unverändert, ihre Reticulumzellen enthalten oft Eisenpigment.

Bekanntlich ist der Pigmentgehalt der Milz bei den einzelnen Tierarten verschieden und von dem Alter der Tiere abhängig [Hueck $\left.{ }^{1}\right)$ ]. Die Milz des Pferdes enthält schon normalerweise bisweilen größere Pigmentmengen [vgl. Mrow ka $\left.{ }^{2}\right)$ ], aber bei der infektiösen Anämie geht die Ablagerung von Eisenpigment weit über das physiologische Maß hinaus und stellt nach meiner Meinung die auffallendste Veränderung dieses Organes bei der chronischen Form der Erkrankung dar (vgl. auch Lührs und Stroh). Nur Mrowka (1.c.) behauptet das Gegenteil und schildert bei der infektiösen Anämie eine Pigmentverarmung der Milz, in der er ein Erschöpfen der Eisenvorräte des Körpers sieht. Demgegenüber konnte ich nur einmal eine Pigmentarmut der Milz konstatieren und das war bei einem Fall, bei dem zwischen letztem Fieberanfall und Tod ein Zeitraum von sechs Monaten lag. Ich glaube, daß während des Fiebers die Erythrocyten geschädigt werden, daß die geschädigten Blutkörperchen sich in der Milz ablagern und hier zu Pigment verarbeitet werden. Später macht sich dann der Organismus dieses Pigment in irgendeiner Form zunutze und wenn lange Zeit keine neuen Fieberattacken erfolgen, so schwindet allmählich das Pigment. Wie werden bei Besprechung der Leber auf diese Frage noch zurückkommen.

Als weiterer Milzbefund bei der infektiösen Anämie wäre die Anhäufung von eosinophilen Zellen hervorzuheben, die auch von anderen Autoren (Seyderhelm Lührs, Stroh, Mrowka) betont wird. Myeloische Elemente waren nur in verstreuten Exemplaren vorhanden, ihre Zahl war zu gering, um von einer myeloischen Umwandlung reden zu können.

Auch bei der perniziösen Anämie des Menschen findet sich in der Milz Phagocytose von roten Blutkörperchen und Siderose [Sternbergs $g^{3}$ ), Ep pinger ${ }^{4}$ ), Ellerman $n^{5}$ ) u. a.), letztere hält sich aber meist in mäßigen Grenzen und ist nie so hochgradig, wie ich sie eben bei der ansteckenden Blutarmut der Pferde geschildert habe. Ferner ist

1) Hueck, Zieglers Beiträge z. allg. Path. u. pathol. Anat. 54, 68. 1912.

2) Mrowka, Zeitsćbr. f. Veterinärk. 31, Heft 2. 1919.

3) Sternberg, Verhandl. der.Deutschen path. Gesellsch. 10, 117. 1907.

4) Eppinger, Die hepato-lienalen Erkrankungen. Berlin 1920.

5) Ellermann, Virchows Archiv f. pathol. Anat. u. Physiol. 228, 247. 1920. 
die Blutverteilung ganz anders. In den akuteren Stadien der Pferdeanämie sind die Sinus erweitert und ganz mit Blut angefüllt, während nach Eppinger (1.c.) bei der perniziösen Anämie des Menschen die von hypertrophischen Endothelien ausgekleideten Sinus nur wenige Zellen enthalten und die Pulpastränge die Ablagerungsstätte der roten Blutkörperchen darstellen. Ich kann aus eigener Erfahrung diese Angaben Eppingers vollkommen bestätigen. Eine Anreicherung eosinophiler Zellen scheint mir auch bei der menschlichen Erkrankung häufig zu sein. Die myeloide Metaplasie ist bei der perniziösen Anämie des Menschen nicht regelmäßig voll ausgebildet und beschränkt sich nicht selten auf einzelne Gruppen von Myelocyten. Die Follikel sind meistens verkleinert.

An der Verarbeitung des Pigmentes nehmen bei der infektiösen Anämie der Pferde die Kupfferschen Sternzellen der Leber lebhaften Anteil. Schon nach den ersten Fieberanfällen sind sie mit großen Pigmentschollen überladen, daneben enthalten sie rote Blutkörperchen, Blutkörperchenschatten, Leukocyten und pyknotische Kernreste. Die mit corpusculären Elementen angefüllten Sternzellen runden sich $a b$ und lösen sich von der Capillarwand los. Für die so außer Funktion gesetzten Wandzellen wird durch Proliferation der erhalten gebliebenen Ersatz geschaffen. Wir sehen dann, wie die wuchernden Sternzellen die Capillarlichtung umsäumen und wie sie zusammen mit den pigmentführenden Zellen das Lumen fast vollkommen verschließen. Die jungen Sternzellen sind rundlich-oval, ihre Kerne sind rund mit zierlicher Chromatinzeichnung, Mitosen finden sich öfters. Das Protoplasma tingiert sich bei Giemsafärbung rötlichviolett und enthält meist schon einige feine Pigmentkörnchen. Diese Zellformen bedingen in erster Linie den Zellreichtum der Capillaren. Daneben bemerkt man rote Blutkörperchen, reichlich eosinophile Leukocyten, bisweilen auch eosinophile Myelocyten, einzelne lymphocytäre Plasmazellen und polymorphkernige Leukocyten.

Die Pigmentzellen finden sich in größerer Zahl auch in den Zentralvenen und in den Verzweigungen der Vena hepatica, in den Ästen der Vena portae sind sie viel seltener. Im periportalen Gewebe sieht man kleine Ansammlungen von Lymphocyten und Plasmazellen. Die Leberzellen sind meistens unverändert und stets frei von eisenhältigem Pigment. Seltener kommen in den mittleren und zentralen Acinusbezirken umschriebene Auflockerungsherde vor. Diese Herde werden von den kollabierten Capillaren durchzogen, zwischen denen die abgerundeten, aus dem Verbande gelösten, hyalin degenerierten oder vakuolisierten Leberzellen liegen.

Wenn die Erkrankung chronisch wird und die Fieberanfälle sich häufen, dann steigern sich immer mehr die Pigmentablagerungen in 
der Leber. Durch das enorme Angebot an geschädigten Erythrocyten ist die Milz funktionell überlastet und die Sternzellen der Leber treten in immer höherem Maße für sie ein. Sie bilden, in lebhafter Vermehrung. begriffen, größere, zusammenhängende Komplexe, die wie ein fremdes Gewebe in das Leberparenchym eingebettet liegen (vgl. Abb. 1 und 2). Die Histogenese dieses Gewebes wird klar, wenn man durch Vergleich der einzelnen Entwicklungsphasen seine Entstehung verfolgt. Die Zellen, aus denen es sich zusammensetzt, sind die gleichen Zellen,

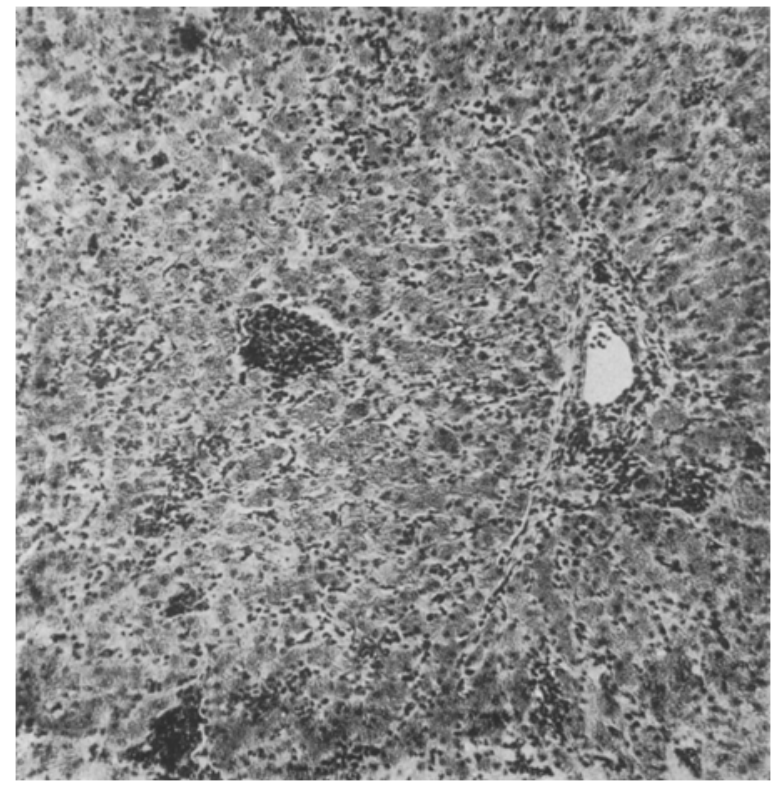

Abb. 1. Formalinfixierung, Gefrierschnitt, Haemalaun-Eosin. Zeiss A. Proj. Ocul. 4.

die wir im akuten Stadium als phagocytierende Sternzellen erkannt haben. Die Phagocyten liegen jetzt so dicht aneinander gepreßt, dab man die einzelnen Zellen nicht mehr voneinander abgrenzen kann (vgl. Abb. 3). Sie verdrängen die angrenzenden Lekerzellbalken und lassen an den Rändern den Übergang in den Wandbelag der Capillaren deutlich erkennen. Die richtige Vorstellung von den Eisenmengen, die die herdförmigen Wucherungen beherbergen, bekommt man auch hier nur bei der Turnbullblaureaktion. Bis auf die verschieden geformten, ausgezerrten, blassen Kerne ist alles mit feineren und gröberen. blauen Körnchen und Schollen überfüllt und manche Zellen färben sich diffus lebhaft blau. Zwischen den Sternzellen fallen bei der Giemsafärbung einzelne große, stark basophile Zellen mit bläschenförmigen Kernen auf. Phagocytose von roten Blutkörperchen ist verhältnis- 


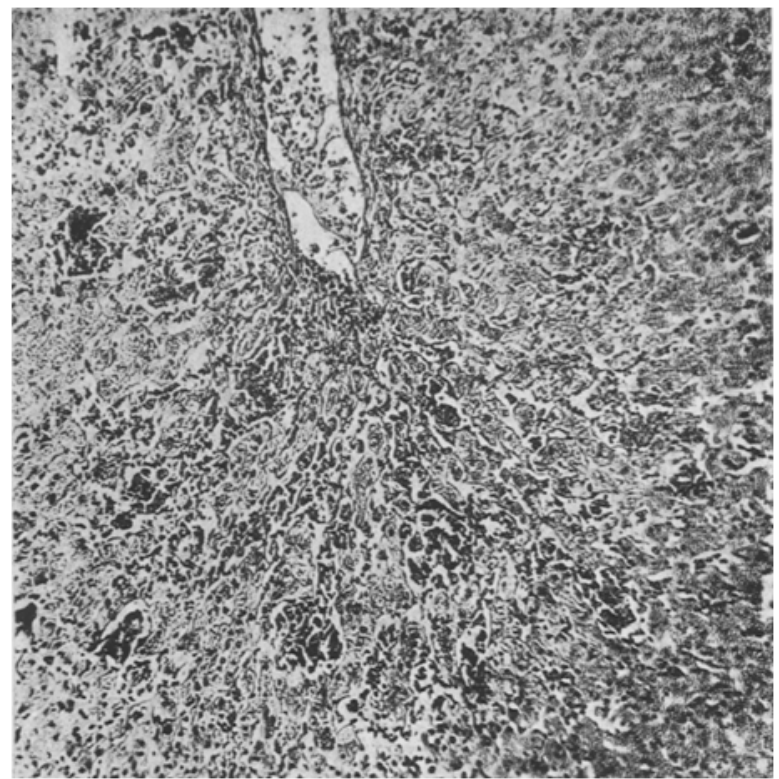

Abb. 2. Alkoholfix, Paraffinschnitt, Haemalaun-Eosin. Zeiss A. Proj. Ocul. 4.

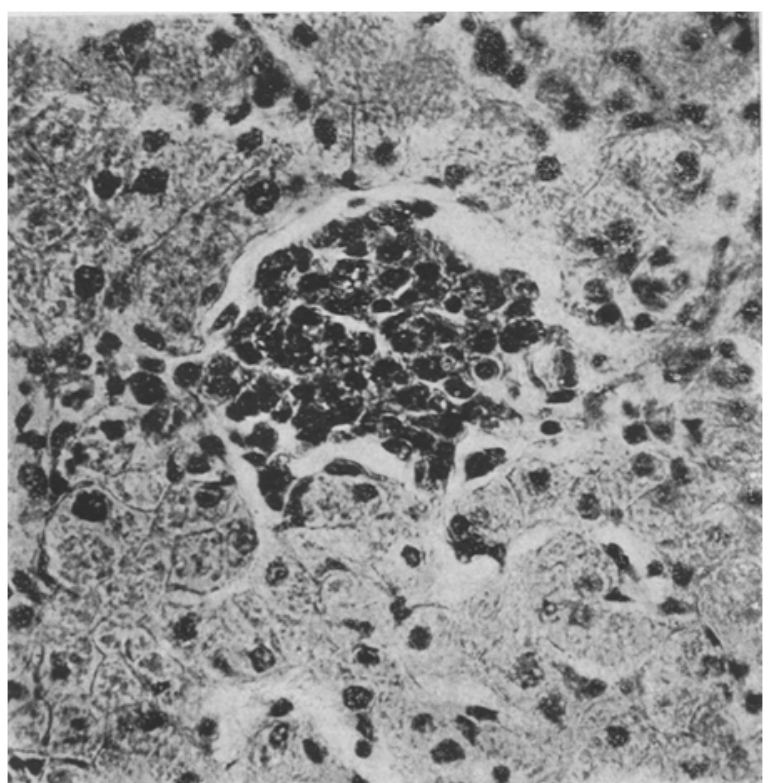

Abb. 3. Alkoholfix., Paraffineinbettung, Haemalann-Eosin. Zeiss D. Proj. Ocul. 4. 
mäßig selten und man muß sich daher vorstellen, daß zum Teil schon in der Milz zu Pigment verarbeitetes Material auf dem Blutwege der Leber zugeführt wird.

Die jüngeren Wucherungsherde bestehen vorwiegend aus lymphoiden Zellen. Von ihnen leiten alle Übergänge zu den großen, pigmentüberladenen Zellhaufen.

An den Leberzellen ist kein pathologischer Befund zu erheben. Vor allem sind sie stets vollkommen frei von siderophilem Pigment. In den zentralen Acinusbezirken finden sich manchmal etwas reichlicher die schon normalerweise in den Leberzellen vorkommenden blaßbraunen, eisenfreien Körnchen. Gallenthromben sah ich nur ganz vereinzelt. Unter den Blutzellen im Innern der Pfortadercapillaren sind wieder in erster Linie eosinophile Leukocyten zu nennen. Das periportale Bindegwebe ist meistens vermehrt und reich an Rundzellen und Plasmazellen, zwischen denen auch pigmentführende Makrophagen vorkommen. Es läßt die Umgrenzung der Leberacini deutlicher hervortreten. Einzelne Leberzellen sind aus dem trabeculären Verbande gelöst und in das gewucherte Bindegwebe verlagert.

Nur einmal unter den zehn untersuchten Fällen vermißte ich den eben geschilderten Befund in der Leber und das war in dem Fall, in dem auch die Milz auffallend arm an Pigment war. Es gilt hier wohl die gleiche Erklärung, die ich für die Milz gegeben habe.

Myeloide Metaplasie der Leber, auf die Seyderhelm und Stroh das größte Gewicht legen, habe ich niemals nachweisen können. Es ist möglich, daß in meinen Fällen die Erkrankung noch nicht jene Grade erreicht hatte, bei denen es zur extramedullären Blutbildung hätte kommen können. In den Beschreibungen von Seyderhelm und Stroh fiel mir auf, daß die myeloiden Herde vorwiegend aus lymphoiden Zelltypen bestanden, die nach Seyderhelm aus proliferierenden Sternzellen hervorgingen. Normoblasten waren viel seltener und vor allem wurden sie in Organabstrichen vermißt (Stroh). Ich habe an anderer Stelle ${ }^{1}$ ) die Argumente zusammengefaßt, die mir gegen einen Übergang von Sternzellen in myeloische Zellen zu sprechen scheinen, and betreffs der lymphoiden Zellformen in meinen Präparaten bin ich zu einer ganz anderen Auffassung gelangt. Läßt schon die fehlende Oxydasereaktion die myeloische Natur dieser Zellen ausschließen, so wird ihre eigentliche Natur bei der Turnbullblaureaktion klar, bei der in ihrem Protoplasma feine Eisengranula sichtbar werden. Daraus ergibt sich, daß sie lymphoide Jugendformen der Sternzellen sind, die ihren Charakter nicht ändern und die auch in diesen Stadien ihre Funktion und ihr weiteres Schicksal erkennen lassen. Zu den Wuche-

1) Zieglers Beiträge z. allg. Path. u. pathol. Anat. 68, Heft 2. 1921. 
rungen der Sternzellen gesellt sich eine Vermehrung des periportalen Bindegewebes, und so werden die Übergänge verständlich, die nach Stroh zwischen der infektiösen Anämie und manchen Formen der equinen Lebercirrhose (Schweinsberger Krankheit) bestehen sollen. (Ửber Blutschädigung und Leberzirrhose, 'vgl. auch Rudolf Jaffé Frankfurt. Zeitschr., 24, Heft 2, 1920.)

Einen wichtigen Unterschied zwischen dem Leberbefund bei der infektiösen Anämie der Pferde und dem bei der perniziösen Anämie der Menschen stellt die Lokalisation des Eisenpigmentes dar. Die Leberzellen der erkrankten Pferde sind frei von Hämosiderin, während sie bei der menschlichen Erkrankung regelmäßig feine siderophile Körnchen enthalten. Die Körnchen liegen anfangs in den peripheren Anteilen der Acini, später verteilen sie sich über alle Läppchenabschnitte und ordnen sich vorwiegend um den Kern. Die Sternzellen nahmen auch bei der perniziösen Anämie des Menschen rote Blutkörperchen und Blutkörperchentrümmer auf und manchmal zeigen sie bei der Turnbullblaureaktion eine diffuse Blaufärbung (Eppinger l. c.). Die Hämosiderinablagerung ist bekanntlich ein direkter Beweis für die Blutgiftwirkung [Paltauf $\left.{ }^{1}\right)$ ]. Die Verteilung des Hämosiderins in der Leber hängt $a b$ von dem Zustand, in dem der Blutfarbstoff angeboten wird. Bekommt die Leber in abnormen Mengen gelösten Blutfarbstoff zugeführt, so schlägt sich derselbe in Form feiner Körnchen in den Leberzellen, Sternzellen und im periportalen Gewebe nieder, gelangen dagegen schollige Blutkörperchenmassen in das Gefäßgebiet der Leber, so beladen sich nur die Sternzellen mit ihnen [Sternberga')]. Bei den durch Pyrogallol und Phenylhydrazin hervorgerufenen Blutzerstörungen sah ich in der Leber nur die Sternzellen Pigment verarbeiten und gleich dem Befunde bei der ansteckenden Blutarmut der Pferde waren die Leberzellen immer pigmentfrei.

Die eigenartigen, aus Sternzellen bestehenden Wucherungsherde in der Leber fehlen bei der menschlichen Erkrankung. Die Wucherungen erinnern in erster Linie an die vikariierende Proliferation der Sternzellen, die M. B. Schmidt ${ }^{3}$ ) bei entmilzten Mäusen beschrieb. Die Pfortadercapillaren der Leber enthalten bei der perniziösen Anämie des Menschen oft Ansammlungen von Knochenmarkszellen, eine extravasculäre myeloide Umwandlung ist dagegen nicht von allen Autoren bestätigt worden (vgl. Ellermann l. c.). Daß myeloide Metaplasie der Leber bei meinen Fällen equiner Anämie fehlte, wurde bereits erwähnt. -

1) Palta uf in Krehl-Marchand, Handbuch der allgemeinen Pathologie. Bd. 2, S. 1. 1912. Bd. 2.

2) Sternberg in Aschoffs Lehrbuch der pathologischen Anatomie. 4. Aufl.

3) M. B. Schmidt, Verhandl. der Deutschen path. Gesellsch. 1\%. 1914. 
Die succulente Schwellung der Lymphknoten beruht auf einer Hyperplasie des lymphadenoiden Gewebes. Die Rindenfollikel sind meistens deutlich abgegrenzt und enthalten große, mitosenreiche Keimzentra. In den erweiterten Sinus liegen Lymphocyten, spärliche rote Blutkörperchen und einzelne desquamierte Endothelien. Die phagocytäre Tätigkeit der Endothelien ist in der Regel geringfügig, nur selten erreicht sie stärkere Grade. Auch Eisenpigment kommt nur in mäßigen Mengen in den Endothelien vor, etwas reichlicher trifft man es in den Reticulumzellen an. Außerdem fällt namentlich bei den chronischen Fällen in den Marksträngen ein schwarzbraunes Pigment auf. Es bildet kleine, kantige Stäbchen, die, von Zellen umschlossen, sich zu dichten Häufchen zusammenfügen. Dieses Pigment ist eisenfrei und erinnert sehr an Malariapigment. Woher es stammt, kann ich nicht entscheiden, vielleicht spricht für seine hämatogene Natur der Umstand, daß es in manchen Zellen neben Erythrocytentrümmern und sicherem Blutpigment liegt.

Einen häufigen Befund bilden ferner auch in den Lymphknoten eosinophile Leukocyten. Sie lokalisieren sich in den Marksträngen und im periglandulären Bindegewebe. Mitunter kann man deutlich ihren Durchtritt durch erweiterte Capillaren feststellen und ich glaube, daß die außerordentlich charakteristischen eosinophilen Leukocyten des Pferdes sehr geeignet sind, um die viel umstrittene Frage ibrer Herkunft bei der lokalen Eosinophilie zu studieren. Die eosinophilen Leukocyten des Pferdes enthalten bekanntlich die größten Granula unter den Granulocyten der Säugetiere (die sogenannten SchmidtSemmerschen Körperchen). Wenn es eine lokale Entstehung der Eosinophilen gibt, so muß sich leicht verfolgen lassen, wie sich die großen, leuchtend roten Kugeln im Innern ungranulierter lymphoider oder histocytärer Vorstufen entwickeln. Davon konnte ich mich aber nie überzeugen und so muß ich sie als aus dem Blute eingeschwemmt auffassen.

Die Rötung der Lymphknoten wird durch eine stärkere Blutfüllung ihrer Gefäße hervorgerufen. Eine Umwandlung in Hämolymphdrüsen kommt nach meiner Erfahrung bei der infektiösen Anämie der Pferde nicht vor und auch myeloide Metaplasie, wie sie bisweilen bei der perniziösen Anämie des Menschen beobachtet wurde, sah ich nie.

Seyderhel m (1. c.) beschrieb bei der infektiösen Anämie der Pferde hochgradige lymphoide Umwandlung des Knochenmarkes. Reinhardt (l. c.), Stroh (l. c.) u. a. schilderten braunrote und dunkelrote Flecke im schwammigen und im Fettmark. Mrowka (1.c.) fand nur Fettmark und Lührs (l. c.) bezeichnete das Knochenmark als nicht charakteristisch verändert. Hutyra und Marek (l. c.) geben an, daß das Fettmark der langen Röhrenknochen ganz oder teilweise in dunkel- 
rote oder schwarzrote Massen verwandelt ist, bei langsamem Verlauf aber auch unverändert befunden werden kann. Die rote Fleckung des Fettmarkes ist nicht nur der infektiösen Anämie der Pferde eigen, sie findet sich auch sonst gelegentlich, namentlich bei „Serumpferden" ist sie häufig. Bei meinen Füllen war auffallenderweise das Knochenmark immer unverändert, die Röhrenknochen enthielten nur reaktionsloses Fettmark und, was makroskopisch als kleinste Blutungen imponierte, waren nur durchschnittene Gefäße.

Aus den mitgeteilten Befunden geht hervor, daß den Knochenmarksveränderungen bei der ansteckenden Blutarmut der Pferde keine ausschlaggebende Bedeutung zukommen kann, denn ihre Ausbildung schwankt in den einzelnen Fällen in weiten Grenzen. Bei der menschlichen perniziösen Anämie wird seit Cohnheims erster Beschreibung in dem roten Knochenmark eine für diese Erkrankung zwar nicht spezifische, so doch für sie sehr charakteristische Veränderung gesehen. Ellermann (l. c.) unterscheidet dabei ein einfach hyperplastisches Mark mit vorherrschenden Myelocyten und ein metaplastisches Mark mit vielen Megaloblasten und großen lymphoiden Zellen (megaloblastische Entartung des Knochenmarks nach Ehrlich).

Über die Nieren finden sich in meinen Aufzeichnungen nur wenige Angaben. Bisweilen enthielten die gewundenen Harnkanälchen zusammengesinterte rote Blutkörperchen, hier und da auch hyaline Zylinder. Die Glomeruli waren unverändert, in ihren Schlingen steckten oft pigmentführende Zellen. Die Epithelien der Harnkanälchen waren frei von Pigment. Ich erwähne dies, weil bei der perniziösen Anämie des Menschen die Epithelien der Tubuli contorti in ihrem lumenwärts gelegenen Anteil feine Eisenkörnchen enthalten, und es sei hier an das über den Pigmentgehalt der Leberzellen Gesagte erinnert.

Über die histologische Untersuchung des Gehirns, der Lungen, des Magens, des Pankreas, der Nebenniere usw. ist nichts zu berichten ${ }^{\mathbb{1}}$ ).

Die Gegenüberstellung der histologischen Befunde zeigt den prinzipiellen Unterschied, der zwischen der infektiösen Anämie der Pferde und der perniziösen Anämie des Menschen besteht und der auch im Blutbilde zum Ausdruck kommt (Wirth l. c., Fröhner l. c.). Damit werden alle Versuche hinfällig, Hypothesen, die zur Erklärung der equinen Anämie aufgestellt wurden (Seyderhelm Stroh), auf die menschliche Pathologie zu übertragen. Auch bei der perniziösen Anämie des Menschen liegt ein toxogener Blutzerfall vor, aber er allein genügt nicht, um den ganzen Krankheitsprozeß zu klären, und immer wieder deuten die Befunde auf das Knochenmark hin, in dessen Erkrankung

$\left.{ }^{1}\right)$ Von allen Organen wurden anch Levaditipräparate angefertigt, Spirochäten wurden in ihnen nicht gefunden. Die Untersuchung des Blutes auf Parasiten (Dunkelfeld usw.) war negativ. 
nach Naegeli der Angelpunkt des Prozesses liegt. Es erübrigt sich, an dieser Stelle die Arbeiten anzuführen, auf denen sich diese Erkenntnis aufbaut. Bei der ansteckenden Blutarmut der Pferde fanden wir eine schwere Schädigung der roten Blutkörperchen während der Fieberanfälle. Die geschädigten Blutzellen wurden von den Retikuloendothelien aufgenommen und weiter verarbeitet. Das Knochenmark bot keine Veränderungen und war in den Kreis der unmittelbaren Folgen der Infektion nicht mit einbezogen. Ich will damit nicht leugnen, daß bei sehr schwerer und langdauernder Blutzerstörung das Knochenmark schließlich auch reagieren kann in der Form, daß es durch gesteigerte Tätigkeit den Blutausfall wettzumachen versucht. Der auf die Retikuloendothelien beschränkte Blutkörperchenabbau erinnert an die Vergiftung mit Pyrogallol, Pyrodin usw. Für manche Infektionskrankheiten des Menschen, ich nenne vor allem den Typhus abdominalis, ist die Phagocytose von roten Blutkörperchen durch Histocyten sehr charakteristisch. An der Hämosiderose in den späteren Stadien des Typhus nehmen auch die Leberzellen lebhaften Anteil. Welcher Art die Schädigung ist, die bei der equinen ansteckenden Anämie die Erythrocyten und wie ich glaube zum Teil auch die Leukocyten trifft, läßt sich aus dem histologischen Bilde nicht erschließen. Es kann aber kein Zweifel darüber bestehen, daß sie infektiöser Natur ist, wie auch aus den neuesten Untersuchungsergebnissen hervorgeht, ich erwähne die Wahrscheinlichkeit der Übertragung durch blutsaugende Insekten (Lührs u. a.) und die gewisse Regelmäßigkeit in der Wiederkehr der Fieberanfälle [Habersang $\left.\left.{ }^{1}\right)\right]$.

1) Habersang, Monatshefte f. prakt. Tierheilk. 32, Heft 1/2. 1921. 